\title{
An Overview of the Primary Cilium and RPGRIP1L: The Signalling Hub's Anchor for Organ Development and Homeostasis
}

\author{
Ivanna Williantarra ${ }^{a, *}$, Timmy Richardo ${ }^{b}$, Inge Kumalasari ${ }^{a}$, Putu Virgina Partha \\ Devanthi ${ }^{\mathrm{a}, *}$
}

a Department of Biotechnology, School of Life Sciences, Indonesia International Institute for Life Sciences, Indonesia; ${ }^{b}$ Department of Biomedicine, School of Life Sciences, Indonesia International Institute for Life Sciences, Indonesia.

\begin{abstract}
Research on the primary cilium has been growing exponentially in the past several decades due to its functions as a cell signalling hub, which defects leads to several disorders and abnormalities collectively known as ciliopathies. Among other parts of the primary cilium structures, the transition zone is the area whose defects lead to the most severe clinical manifestations and high lethality. The ciliary transition zone consists of multiple protein modules that are hypothesized to be anchored by the RPGRIP1L protein. Despite its importance, RPGRIP1L studies remain hidden from the limelight, and our understanding of the protein remains scattered. This review summarizes the clinical manifestations and molecular mechanisms of the RPGRIP1L in the primary cilium. We then take a closer look at each RPGRIP1L's protein domain to understand how each domain ensures proper functions and localization of RPGRIP1L. The three domains of RPGRIP1L are postulated to be involved in different roles. While the coiled coil domain is vital for scaffolding the protein to the centriolar structure, the ability of the $\mathrm{C} 2$ domain to interact with lipid allows the formation of 'lipid gate' at the transition zone. The high variability of the RPGR interaction domain enable the RPGRIP1L to interact with multiple different proteins, making it an ideal anchor for other ciliary protein modules in the transition zone.
\end{abstract}

Keywords: cilia ciliogenesis, ciliopathies, primary cilia, RPGRIP1L, transition zone

*For correspondence: i.williantarra@auckland.ac. $\mathrm{nz}$

Received: 6 June 2021

Accepted: 11 October 2021

(C) Copyright Williantarra.

This article is distributed under the terms of the Creative Commons Attribution License, which permits unrestricted use and redistribution provided that the original author and source are credited.

\section{Introduction}

Primary cilium serves as a cellular antenna that mediates signals for many signalling pathways, including Hedgehog, Platelet-Derived Growth Factor (PDGF), and Wnt [1]-[4]. Not surprisingly, defects in primary cilia are linked to several disorders collectively known as ciliopathies, including congenital disabilities, bone and brain abnormalities, cyst formation in organs, and cognitive disorders [5], [6]. Many mutated proteins in ciliopathies are localized at the transition zone, a compartment at the primary cilium's proximal end. The loss of the transition zone compartment can disrupt ciliary functions such as the control of protein trafficking in and out of the cilium, the regulation of signalling cascades, and control of the cell cycle [7]. Therefore, understanding the functional modules and pleiotropic within the transition zone is essential to find a cure for various ciliopathies disorders.

RPGRIP1L (retinitis pigmentosa GTPase regulator-interacting protein 1-like) is one of the transition zone proteins known to anchor another ciliary protein. It is, therefore, explanatory that missing RPGRIP1L will lead to lethal diseases, and most of the patients do not survive post-natally. In this review, we first emphasize primary cilia types and structures with a special focus on the cilia transition zone. We then elaborate on the molecular signature of the RPGRIP1L in correlation to ciliopathy diseases. 


\section{Primary cilia in a nutshell}

Long considered a vestigial organelle, primary cilia is now well known as a cell signalling hub. The recent excitement of primary cilia research was somewhat sluggish in the past due to a limitation in microscopy resolution. Being a non-motile organelle smaller than $10 \mu \mathrm{m}$ [8] makes research on primary cilia only started to emerge in the 1950s after the invention of electron microscopy [9].

In contrast to primary cilia, motile cilia have been studied in detail due to their readily observable nature in cell motility. The motile cilium is the oldest known organelle, first described by Leuwenhoek in 1674 . Motile cilia and flagella are the two cell motors that share a similar internal structure of having nine doublet tubules on the perimeter and an addition of another doublet in the centre, thereby making a $9+2$ pattern (Fig. 1) [10]. The tubules, or so-called the axonemes, are an extension of the centrioles enclosed by protein-rich membranes. Many protein complexes interconnect within the microtubule core; one of which is the outer and inner dynein arm that attaches on the periphery microtubule and slides the doublet tubules within one another. This ATP-dependent sliding occurs in certain periodicity and produces a force that leads to beating movement that powers the cell movement [4], [11].

Although cilia and flagella are essentially the same, they were given different names because their structure has been thoroughly studied. Flagella would usually adopt a more extended structure that can exceed $2 \mathrm{~mm}$, as demonstrated in some insects' sperm flagella, while cilium is only 1-10 $\mu \mathrm{m}$ in length. The numbers of flagella on a cell are typically about one to eight, much fewer compared to cilia, which can exist in hundreds [12].

For a very long time, ciliary research only focused on motile cilia. Only two centuries after cilia discovery, Alexander Kowalevsky became the first to describe the presence of non-motile cilia in 1867. Kowalevsky found that in contrast to motile cilia, non-motile cilia is solitary and widely distributed in almost all vertebrate cells [13]. Not much progress was made after since the non-motile cilia were thought to have no real role. In 1962, Sorokin did an electron microscopy study and elegantly revealed the process of non-motile cilia formation or ciliogenesis. Sorokin's study was also the first to use the term primary cilia for the non-motile cilia [14].

Many electron microscopy studies were conducted to learn the structure of primary cilia following Sorokin's study [15], [16]. Both primary and motile cilia are microtubule-based, membrane-bounded, and centriole derived structure [17]. However, while motile cilia have the $9+2$ pattern, primary cilia have a $9+0$ due to the absence of the central pair. The central pair acts as a scaffold for the complex, and the lack of such structure consequently results in loss of motility. Adams [18] investigated the significance of the central pair using Chlamydomonas reinhardtii as a model organism. He mutated several central pair complex genes, which eventually resulted in paralyzed rigid flagella. Other than the microtubule pattern, primary cilia also have no nexin link and dynein arms, vital for motility [17], [19].

The opportunistic nature of evolution has enabled specific structures to modify as a means of adaptation. Modified cilia can be found from lower animal classes to the higher vertebrates, with the association to sensory functions being the most prominent. The mammal's eye rods and cones outer segments are expanded 9+0 cilia. Olfactory cells have incredibly long non-motile chemoreceptive cilia (up to $200 \mu \mathrm{m}$ ), which are $9+2$ at the base but gradually lose this organization distally. Nodal cilia are propeller-like cilia with a 9+0 pattern found in the gastrulation stage embryo [12], [17], [20]. It is noteworthy that most of the knowledge about primary cilia nowadays is based on studies conducted in lower animals such as Tetrahymena, Chlamydomonas, and Caenorhabditis elegans. Nevertheless, a proteomic study by Ostrowski [21] shows the presence of human orthologs of virtually all cilia protein machinery marking the conserveness of cilia despite many modifications available.

As the structure of primary cilia is more investigated, scientists started to hypothesize primary cilia's function. Interestingly, scientists found that primary cilia are only present when the cells are in their quiescent state, which leads them to question the significance of primary cilia [22]. For this reason, scientists initially believe that cilia have no role that is now known to be untrue. Defects in primary cilia can lead to ciliopathies that can manifest in a constellation of severe clinical features such as retinal 
degeneration and cerebral anomalies with high lethality [5], [23].

Another hypothesis of primary cilia's functional significance is its role as a centriole sequestering agent [4], [24]. The fact that primary cilia are centriole derived and exclusively available only when the cells are in the G1 phase makes scientists think that primary cilia might inhibit cell division. This hypothesis is somewhat correct because when the cells leave the G1 phase, primary cilia will start to disassemble only to re-emanate in the daughter cells once they enter the quiescent state [22].

The third and now proven as the most accurate hypothesis is the primary cilia as the cell antenna. Many experiments show that the ciliary membrane is abundant in ion channels, receptors, and transport proteins, contributing to the primary cilia's capability as a cellular sensory structure [25]. The presence of many sensory proteins enables cilia to initiate a cascade of downstream signalling processes leading to gene expression and cellular responses. Hedgehog signalling is one of the primary cilia signalling, which has been progressively studied in addition to several others, such as the Wnt signalling, mechanotransduction, and cell polarity [26].

The momentous turnover of primary cilia research comes from its counterpart, the flagella. Dozens of researchers had studied the structure and functions of flagella in Chlamydomonas as a model, but no one knew how the structure was assembled. In 1993, intraflagellar transport (IFT) was discovered, giving a hint to the problem [27]. Later in 1998, a study by Nonaka [18] reveals that IFT is also found in cilia, followed by another twig finding at the turn of the century, which confirms the association between IFT and polycystic kidney disease (PKD), the most prevalent kidney genetic disease in human due to defects in primary cilia assembly [28]. The connection between primary cilia and pathologies was established instantly.

By the 2000s, primary cilia have moved from obscurity to vigorous research efforts in the cellular and molecular biology field. The technological innovation such as the super-resolution microscopy and omic studies has enabled us to understand both the structure and function of this once underappreciated organelle. Although research progress has been rapid, there are still many unexplained phenotypes in ciliopathies manifestation, which opens the possibility of primary cilia's unexplored roles. Table 1 summarizes key discoveries in primary cilia research.

Table 1. Key discoveries and events in primary cilia research

\begin{tabular}{ll}
\hline Year & Milestone \\
\hline 1674 & Leeuwenhoek discovered motile cilia \\
\hline 1867 & $\begin{array}{l}\text { Alexander Kowalevsky first described non-motile cilia as solitary and widely distributed organelle in almost } \\
\text { all vertebrate cells }\end{array}$ \\
\hline 1950 & Studies on cilia started to emerge \\
\hline 1962 & $\begin{array}{l}\text { Sorokin identified the process of non-motile cilia formation or ciliogenesis. The term primary cilia for the } \\
\text { non-motile cilia was used for the first time. }\end{array}$ \\
\hline 1980 & The ultrastructure of primary cilia in quiescent 3T3 cells was investigated \\
\hline 1985 & Morphology and function of primary cilia in connective tissues were described \\
\hline 1993 & Intraflagellar transport (IFT) was discovered in green alga Chlamydomonas \\
\hline 2000 & IFT was found in cilia \\
\hline 2002 & IFT's role for primary cilia assembly in mammals and its correlation with polycystic kidney disease (PKD) \\
\hline 2007 & were identified \\
\hline 2011 & First proteomic analysis of the human ciliary axoneme \\
\hline 2015 & Mutation in RPGRIP1L gene was identified in patients with ciliopathies caused by defective primary cilia \\
\hline
\end{tabular}

\section{Primary cilia structure}

The cilium is an extension of a mother centriole, which serves as the axoneme nucleation centre. When the cells are not in the G1 phase, the centriole is located adjacent to the nucleus, surrounded by a cloud of the pericentriolar matrix (PCM), altogether known as the centrosome [29]. One essential protein in the $\mathrm{PCM}$ is the $\mathrm{y}$-tubulin, which is now widely used as the centrosome marker. Upon cell entry to the 
quiescent state, the centrosome migrates to the cell apical surface to mature as the basal body. The basal body's docking at the cell surface is facilitated by the subdistal appendage, a specialized structure at the central part of the mother centriole cylinder [30], [31]. The docking is hypothesized to be further stabilized by the striated rootlets, a collection of fibres projecting out from the basal body's proximal end [32].

The axoneme is protruded out from the basal body and bounded by the ciliary membrane. The ciliary membrane is not a separated structure but rather a continuation of the plasma membrane [17]. At the root of the cilia, a specialized domain called the ciliary pocket facilitates endocytic transport, vesicular trafficking, and the actin network of the cilia [33]. The overall ciliary membrane is relatively much more abundant in proteins compared to another region of the plasma membrane, making scientists hypothesize the existence of a 'gating mechanism' at the base of primary cilia, which promotes selective protein transport into and out of cilia. This selection is hypothesized to be provided by the distal appendage and the transition zone [34]

The distal appendage, also known as the transition fibres or alar sheets, is located at the proximo-distal axis of the mother centriole and assumed to have a similar role as the nuclear pore complex; it promotes size-dependent protein selection [29], [30]. At the distal end of the distal appendage, the transition zone proteins are highly organized to perform another permeability barrier of the cilia. The transition zone is an intricate structure consisting of different protein modules aligned on the $Y$-shape linker. The transition zone is crucial that a mutation in its proteins leads to various ciliopathies diseases [17], [30], [35].

Proteins that could pass the ciliary gating mechanisms provided by the transition zone would then be transported on the cilia axoneme by IFT. Two motor proteins facilitate the IFT; kinesin is the anterograde motor, which walks to the growing (+) end of microtubule and cytoplasmic dynein, the retrograde motor, which paces to the opposite direction [36]. The cargo docking happens at the distal appendage, where the proteins will be docked to the kinesin- 2 motor protein family along with the IFT subcomplex A and B to be transported to the ciliary tip. Not only IFT mediates protein transport essential to relay signalling cascade, but it also enables the assembly and resorption of cilia [34]. Fig. 1 shows a simplified schematic structure of primary cilia.

\section{The ciliary transition zone}

The transition zone originates at the basal body's distal end and constitutes the proximal end of the primary cilium. The transition zone is an intricate structure consisting of different protein modules that are thought to mediate basal body-ciliary membrane anchorage, establishing membrane diffusion barrier and ciliary protein gating [7], [37]. As it fosters multiple functions in the building of primary cilia, many ciliopathies are associated with defects in transition zone proteins [17], [30], [35].

The transition zone encompasses a propeller-like array called transitional fibres at its most proximal end (see Fig. 1 for illustration). Transitional fibres projected out from the B tubules of the basal body just before $C$ tubules termination. Transitional fibres are thought to anchor the basal body to the ciliary membrane [38] through CEP164 [39] and ODF2/cenexin [40] proteins, although not much of its composition is known. The presence of IFT52 has also been reported in Chlamydomonas, suggesting transitional fibre's role in ciliary protein transport [41].

The transition zone's main body is characterized by multiple rows of Y-shaped linkers attaching to the ciliary membrane. The association of these linkers to the membrane forms a circumferential membrane structure referred to as the ciliary necklace. The linkers' detailed protein structure is yet characterized, but it is assumed to connect the outer doublet of microtubules to the ciliary membrane [42], [43]. CEP290 in Chlamydomonas [44] and RPGRIP1L in C.elegans [45] have been reported to localize in the ciliary necklace. Like the transitional fibres, this region is also proposed to facilitate ciliary protein trafficking, although the detailed mechanism remains elusive. The transition zone ends with the last row of $Y$-linkers. 


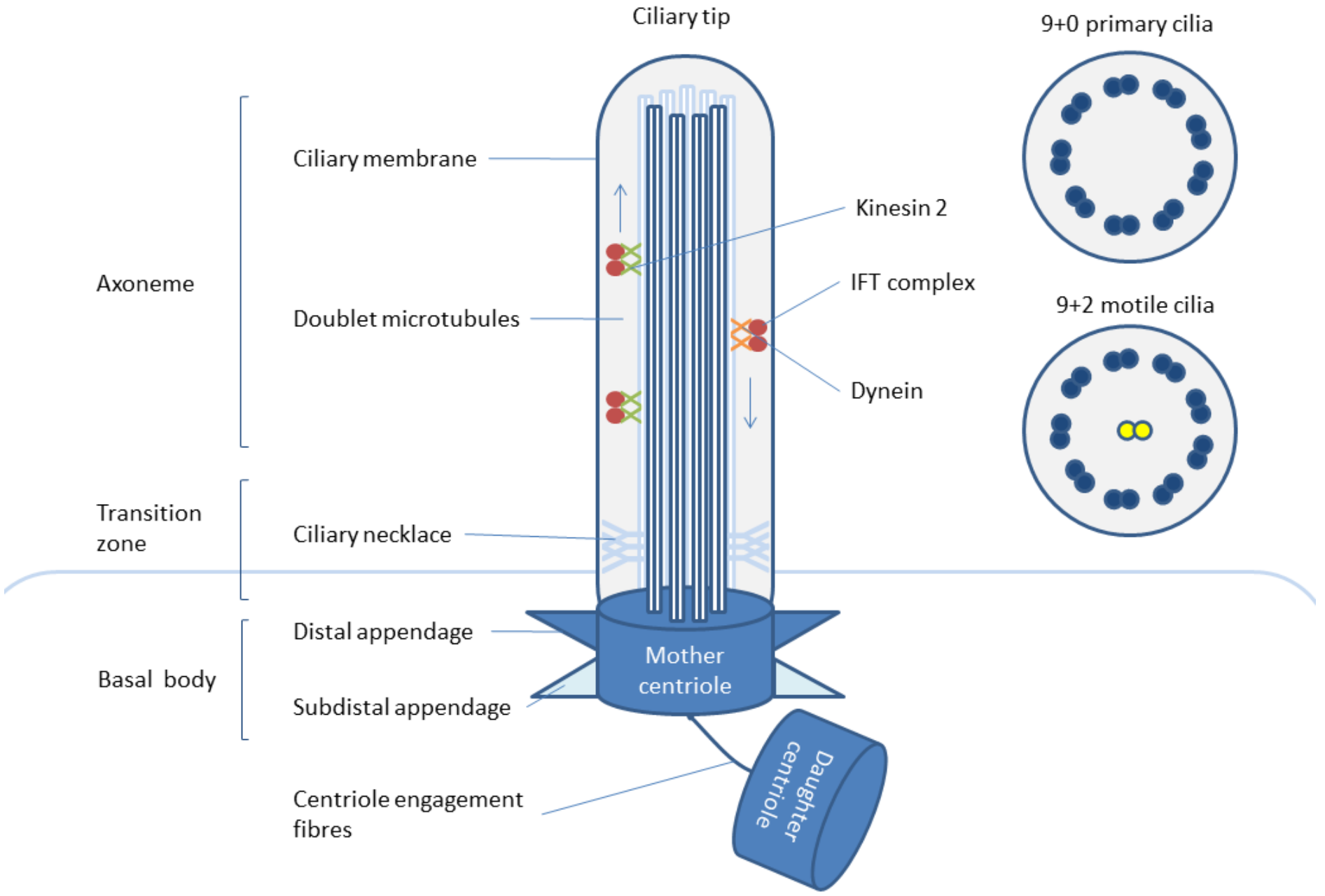

Figure 1. A simplified schematic structure of primary cilia and the cross-sections of cilia axoneme. The size of each compartment is not to scale.

The transition zone consists of at least three different but interconnected protein modules; the NPHP (nephronophthisis), JBTS (Joubert syndrome), and MKS (Meckel-Gruber syndrome) modules [7]. The NPHP module is composed of NPHP1, NPHP4, and RPGRIP1L. This module is suggested to regulate cargo and IFT entry. The second module, the JBTS, contains IQCB1 and CEP290, while the third module, the MKS, comprises MKS1, CC2D2A, and TCTN2, and is essential in Hedgehog signalling. The NPHP/JBTS/MKS are bridged by the inversin (INVS) and AHI1/Jouberin activity [46]. Although the transition zone module proteins are located at the base of the primary cilium, a recent study by Gogendeau [47] showed that the transition zone modules control ciliary shedding at its distal end, which opens a new direction for understanding cilia physiology.

Many studies have shown that transition zone proteins are recruited hierarchically. Retinitis pigmentosa GTPase Regulator 1 Like (RPGRIP1L), previously known as KIAA1005, serves as the anchor of other transition zone protein modules. Located on chromosome 16q12.2, the RPGRIP1L gene encodes 1315 amino acids with a molecular mass of $151 \mathrm{kDa}$ [48]. RPGRIP1L shares $31 \%$ identity and $50 \%$ similarity with its homolog RPGRIP1 but localize at different sub-ciliary regions; while RPGRIP1 localize to the basal body, RPGRIP1L localize to the transition zone [49]. Unlike its homolog RPGRIP1, which is exclusively present in the retina, RPGRIP1L is also located in other tissues such as the brain and kidney [48], [50]. During mitosis, RPGRIP1L resides at the centrosome and migrates to the transition zone of primary cilia during the $\mathrm{G} 0$ phase [51]. 
RPGRIP1L anchors both MKS and NPHP modules in C.elegans. The two modules are then responsible for endogenous localization to the transition zone for other investigated proteins [45]. Huang [52] confirms this as he showed that TMEM237 requires RPGRIP1L for transition zone localization in both IMCD3 and C.elegans. The NPHP/JBTS/MKS modules have also been shown to recruit ATXN2 [46] and TCTN1 [53]. The hierarchy of transition zone protein recruitment is shown in Fig. 2. There is an inconsistency among studies possibly caused by different structures and dynamics of ciliogenesis between tissues. As RPGRIPL is evidently essential for proper recruitment of other ciliary transition zone proteins, defect in RPGRIP1L is associated with multiple developmental disorders including craniofacial development, patterning of the limbs, and formation of the left-right axis, which will be entailed in the following session.

\section{Clinical manifestations of RPGRIP1L associated disorders}

In 2007, Delous et al. [49] were the first to report that RPGRIP1L mutation can lead to severe clinical manifestations. He found that missense and truncating mutations of RPGRIP1L can cause the multiorgan phenotypic abnormalities found in developmental autosomal recessive disorders Joubert syndrome type B (JBTS) and Meckel syndrome (MKS). JBTS is an autosomal recessive multisystem disease characterized by cerebellar ataxia, developmental delay, hypotonia, irregular breathing pattern, eye movement abnormalities, and cerebral vermis hypoplasia dysplasia accompanying brainstem defects. MKS is an autosomal recessive lethal condition characterized by malformation of the central nervous system, postaxial polydactyly, cystic kidney dysplasia, and ductal proliferation in the liver portal area. To date, RPGRIP1L mutations have been reported to cause developmental defects of multiple organs, including brain, heart, eyes, kidneys, lung, liver, skin, and limbs [49], [50] [54]. Table 2 enlists several syndromes associated with RPGRIP1L mutation in the corresponding organs.

The importance of RPGRIP1L in cellular processes is mostly investigated using animal models. A knockout study using a mouse model revealed that the absence of RPGRIP1 in photoreceptor cells resulted in early retinal degeneration, with most of the photoreceptors degenerating by three months of age, marked by the disorganized outer segments as a sign of the cell death process [55]. The mice presented a typical connecting cilia structure, indicating that RPGRIP1 is not necessary for connecting cilia development and maintenance. RPGRIP1 is also essential for normal rod photoreceptor outer segment elaboration and morphogenesis [56].

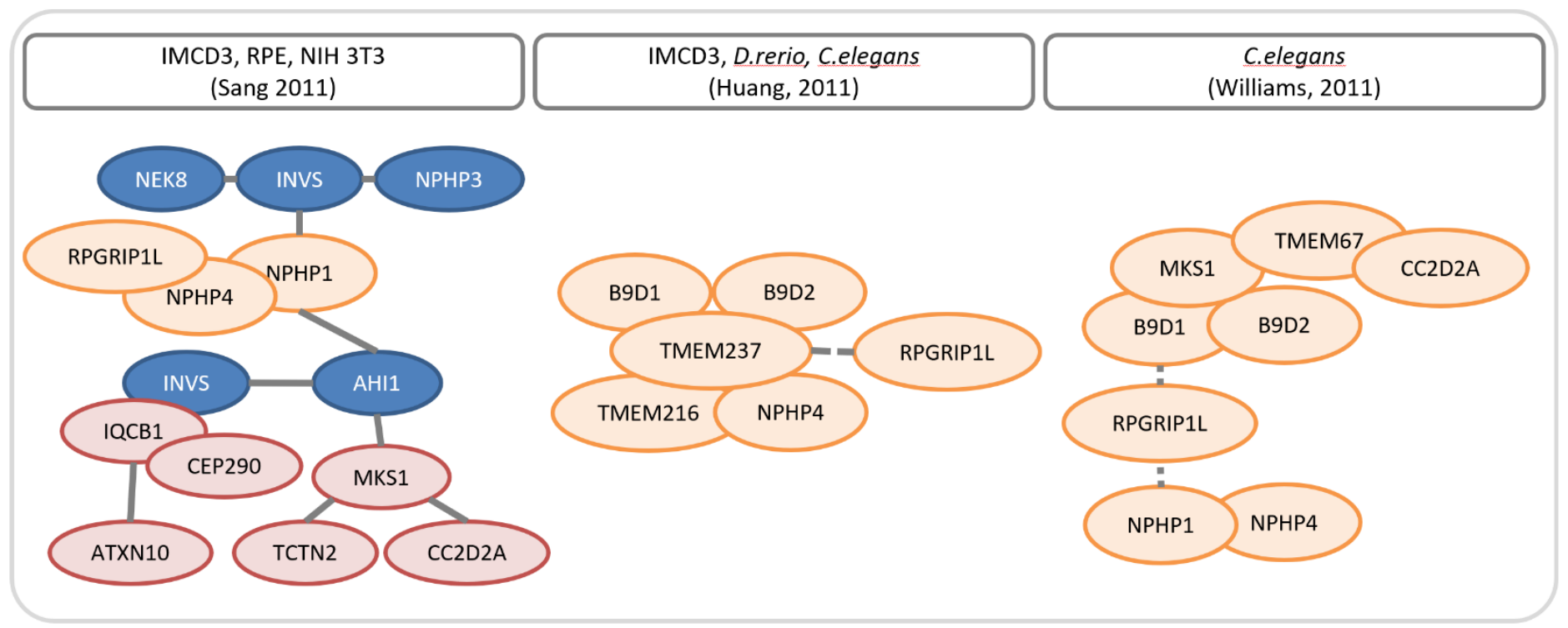

Figure 2. Localization and recruitment of transition zone proteins to sub-ciliary compartments (orange, transition zone; blue, inversin compartment; red, basal body). Overlapping ellipses indicate direct interaction, dark lines indicate interaction identified by mass spectrometry, dashed lines indicate genetic interaction (adapted from [7]). 
Table 2. Syndromes associated with RPGRIP1L mutation.

\begin{tabular}{llc}
\hline Syndromes of RPGRIP1L mutation & \multicolumn{1}{c}{ Organs/system organ affected } & Reference \\
\hline Meckel syndrome (MKS) & Limbs, kidney, central nervous system & [46], [47], [57] \\
Joubert syndrome (JBTS) & Central nervous system, kidney, eyes, limbs & [57] [59] \\
$\begin{array}{l}\text { Cerebellar vermis aplasia oligophrenia } \\
\text { congenital ataxia coloboma hepatic }\end{array}$ & Liver, central nervous system & \\
fibrosis (COACH) & & \\
Nephronophtisis (NPHP) & Kidney & {$[46],[58]$} \\
Bardet biedly syndrome (BBS) & Kidney, limbs, eye, endocrine & {$[58],[62]$} \\
Retinitis pigmentosa (RP) & Eye & {$[63],[64]$} \\
Leber's congenital amaurosis (LCA) & Eye & {$[58],[65]$} \\
Multiple primary tumours (MPT) & Multiple organs & {$[59]$} \\
\hline
\end{tabular}

RPGRIP1L has also been implicated in neuropsychiatric disorders. Injection of phencyclidine, an NMDAR (N-methyl-D-aspartate receptor) antagonist in nerve cells, leads to shortening of the primary cilium and downregulation of RPGRIP1L and other primary cilium related genes [66]. Furthermore, a genome-wide associated study (GWAS) also used RPGRIP1L marker to identify schizophrenia and bipolar disorder [67], [68]. Not only in animal studies but DNA variant in the RPGRIP1L gene in human postmortem brain tissue has also been demonstrated to influence alternative splicing associated with bipolar disorder and schizophrenia [69], [70].

Another animal study using zebrafish demonstrated that morpholino knockdown of rpgrip1/ leads to a shortened body axis, malformed somites, curved body and notochord, and abnormal tail elongation. RPGRIP1L drives motile cilia polar positioning on the floor plate and stabilizes the Dishevelled protein (Dvl) at the cilia base [71]. Inactivation of the rpgrip1/ (ftm) gene leads to the premature death of mice at midgestation, which is almost similar to a malformation of several organs such as brain, liver, kidney, limb, and eye development defects in the human fetus [55], [72].

How defect in RPGRIP1L precisely leads to severe manifestations is still an ongoing research topic. However, an increasing body of work has shown that RPGRIP1L is involved in at least three mechanisms: (1) transition zone assembly, (2) proteasomal activity at the ciliary base, and (3) autophagy via the mTOR signalling pathway.

\section{RPGRIP1L is essential for transition zone assembly}

The genetics of transition zone assembly has been extensively studied using mutations, knockout, and RNAi knockdown [44], [53], [73], [74]. These studies revealed hierarchical patterns and in some cases, ultrastructural correlations of transition zone assemblies and further complemented by systematic analysis on the proteomic of affinity-purified protein complexes using various transition zone proteins as 'bait' [46], [53], [75]or through the direct enrichment of ciliary material [76], [77].

In C.elegans, RPGRIP1L has been shown to occupy a top position in the stringent transition zone assembly hierarchy. Further investigations exposed the role of RPGRIP1L in establishing a ciliary zone of exclusion (CIZE), which confines signalling proteins to distal ciliary subdomains [45], [78]. RPGRIP1L also demonstrates a conserve reciprocal interaction with NPHP1 and NPHP4 [77]; it controls the localization of both proteins and the entire MKS module. Mutations on the NPHP1 and NPHP4 yielded no significant effect on the MKS module, but when mutated altogether, the Y-linkers and membrane attachment were lost entirely, resulting in defective ciliogenesis. Given the top hierarchical position of RPGRIP1L, it explains why the clinical manifestations of RPGRIP1L mutation are much more severe compared to a mutation on other transition zone proteins.

Not only in invertebrate C.elegans, RPGRIP1L also plays a pivotal role in the development of murine cells. In murine cells, the presence of RPGRIP1L drives the amount of MKS module members. RPGRIP1L collaborates with its homolog, RPGRIP1, to warrant proper transition zone assembly [56], [79]. However, the underlying mechanism of RPGRIP1L and RPGRIP1 synergistic control transition zone 
assembly has to be elucidated in the future.

\section{RPGRIP1L regulates proteasomal activity at the ciliary base}

Cell viability heavily depends on the balance between protein synthesis and degradation, and many studies have indicated the association of primary cilia in such a role, specifically in the ubiquitinproteasome system (UPS) and autophagy.

The UPS is a eukaryotic protein degradation system wherein the proteins targeted for degradation are tagged with a multimer protein, the ubiquitin, to be recognized by the proteasome degradation complex. A study by Liu [80] demonstrates that basal body proteins regulate proteasome activity. Moreover, many proteasome proteins are located in the basal body; some even interact directly with the transition zone protein RPGRIP1L [51]. Inhibition of proteasome activity by pharmacological approach causes the cilia to extend significantly.

Multiple studies using mouse embryos have demonstrated the essential organ-dependent role of RPGRIP1L in hedgehog signalling. Further investigation concluded that the absence of RPGRIP1L impaired the proteolytic processing of the Gli3 protein, one of the primary mediators in the hedgehog pathway, by turning it into a transcriptional repressor [81]-[83]. The knockout of rpgrip1/ in mice did not alter the overall cellular proteasomal activity, but it exclusively reduces the proteasomal activity at the ciliary base, which implies a particular proteasome activity at the primary cilium. Further investigation unravelled that RPGRIP1L interacts with 26S proteasome non-ATPase regulatory subunit 2 (Psmd2) protein [51].

Since UPS is a global protein degradation system, its regulation is most likely related to the cell cycle. One possible linker among the three systems is the trichoplein or mitostatin. Trichoplein negatively regulates ciliogenesis by activating AURKA (Aurora Kinase A), a ciliary disassembly protein. Upon G1 phase entry, trichoplein is degraded by the UPS, allowing ciliogenesis to take place [84], [85]. It is noteworthy that ubiquitination does not necessarily lead to degradation as it also regulates many other different pathways, which raises more possibilities for the ubiquitination system to encroach on ciliogenesis.

\section{RPGRIP1L drives cellular autophagy via mTOR signalling pathway}

Autophagy is another type of protein degradation system which mediates non-selective degradation to ensure amino acid turnover in stress condition. Autophagy has been shown to regulate ciliogenesis. The degradation of OFD1, a centriolar protein that needs to be removed to initiate ciliogenesis, is impaired in autophagy mutant, prompting ciliogenesis inhibition [86]. More evidence also reveals the correlation between autophagy and IFT, although the correlation is anything but straightforward; whereas autophagy eliminates IFT machinery, some IFT proteins are proven to regulate autophagy positively [87], which indicates that IFT and autophagy regulate each other although the exact mechanism deserves a further investigation.

The absence of RPGRIP1L is associated with reduced autophagic activity independent of its proteasome-regulating function [88]. Instead, the absence of RPGRIP1L triggers the activation of ciliamediated Mechanistic Target Of Rapamycin Kinase (mTOR) signalling, which inhibits autophagy. mTOR is a conserved pathway that controls cell metabolism and growth. mTOR collaborates with the degradation system to maintain the protein balance inside the cell. High activity of mTOR will constrain autophagy; hence more ciliary protein will be synthesized, but less protein will be degraded and ultimately lead to longer cilia [89], [90]. Therefore, the two systems negatively impact ciliogenesis; mTOR promotes ciliogenesis while the degradation system ensures the opposite. Upon treatment of Rpgrip1/-negative mouse embryonic fibroblasts with an mTOR inhibitor, rapamycin, autophagic activity was restored, and cilia length alteration was rescued [88], [91].

It is noteworthy that UPS and autophagy are correlated reciprocally during ciliogenesis and cilia length maintenance. Proteasomal machinery is regulated by autophagy mechanism (proteaphagy), while key components of autophagy may also be degraded by UPS system. Although more research efforts need 
to be done to fill the knowledge gap in cilia proteostasis, our current understanding indicates a highly orchestrated intersection between proteostasis and cilia. One plausible explanation would be that cilia integrate numerous inputs and mediate the overall cellular process through the proteostasis processes.

\section{RPGRIP1L structure and domain prediction}

Considering its importance in transition zone assembly, proteasomal activity and autophagy, it is essential for RPGRIP1L to function correctly. RPGRIP1L has a relatively big size, enabling direct interaction with many proteins due to its high surface area. Moreover, our unpublished data of 3D analysis using DeepView software (Fig. 3) shows that RPGRIP1L has lots of disordered regions (depicted as thin strings), which enhance its flexibility in interacting with other proteins. The disordered regions remarkably increase the accessibility of RPGRIP1L, thus enabling multiple interactions with other proteins. Its flexibility also allows RPGRIP1L to have numerous molecular functions.

The vital role of RPGRIP1L as a binding agent to recruit other proteins makes it essential for the protein to be able to localize correctly. Recently, RPGRIP1L is also predicted to have a role in tethering the axonemal microtubules to the ciliary membrane [47]. Nevertheless, research on such fields remains elusive. Many studies have demonstrated the presence of signal peptides, which signals the protein to be transported to its cellular destination. The amino acid sequence consensus for such peptides has been thoroughly studied for many organelles such as the nucleus, mitochondrial, chlorophyll, but not for primary cilia. The signalling peptide can be found in various amino acid positions, but they are generally accessible after 3D folding since they need to bind to a peptidase, which will sort the proteins before being transported [92].

As also shown in Fig. 3, RPGRIP1L harbours three distinct domains, separated by intrinsically disordered regions. Those three domains are the coiled-coil domain located on the $\mathrm{N}$-terminal with two leucine zipper motif (red), a conserved region in the middle of the protein containing two protein kinase (C2) (yellow) and RPGR interacting domain (RID) on the C-terminal as the least conserved region (green). Remarkably, each domain shows a distinct function when analyzed separately. When we tried to analyze the protein's signalling peptide, the prediction tool (phobius.sbc.su.se) reveals RPGRIP1L's noncytoplasmic nature but fails to predict the signalling peptide. We tried several other prediction tools such as TargetP, PredSignal, and SignalP, but none of the tools could predict the signal peptide, which means no consensus has been settled for ciliary localization. Although no signal peptide consensus was found, the $\mathrm{N}$-terminal coiled-coil domain is invariant in sequence and length. Moreover, centrosomes are abundant in coiled-coil proteins, which appear to be molecular spacers whose lengths dictate the evolutionarily conserved ninefold symmetric centriolar structure. Therefore, despite the absence of signal peptide consensus, we hypothesized that the coiled-coil domain plays a key role in forming symmetric assembly of radiating spokes required for centriolar localization.

While the coiled-coil domain drives RPGRIP1L localization, C2 domain might play a role in establishing the CIZE lipid gate. C2 domain is a $\mathrm{Ca}^{2+}$-dependent membrane-targeting module found in many cellular proteins involved in signal transduction or membrane trafficking. Its unique membrane targeting property is contributed by its wide range of lipid selectivity, including phosphatidylserine and phosphatidylcholine. C2 domain's unique lipid interaction allows the building of CIZE lipid gate by restricting phosphatidylinositol 4,5-bisphosphate (PIP2) [78]. That C2 domain regulates lipid interaction also explains how RPGRIP1L affects adipogenesis and adipocytes biology, leading to obesity [93], [94].

As the name suggests, the C-terminal RPGR interacting domain (RID) is predicted to accommodate protein-protein interaction. As the least conserved region, RID is also a C2 domain, but no evidence of $\mathrm{Ca}^{2+}$ and/or phospholipids bindings have been reported. As a specialized C2 domain, RID constitutes a unique protein-protein interaction module that might be cilia-specific in multiprotein transition zone complexes [95]. The RID, together with the intrinsically disordered regions, enables interactions between RPGRIP1L and multiple other proteins, which is essential for its function as the transition zone molecular anchor. 


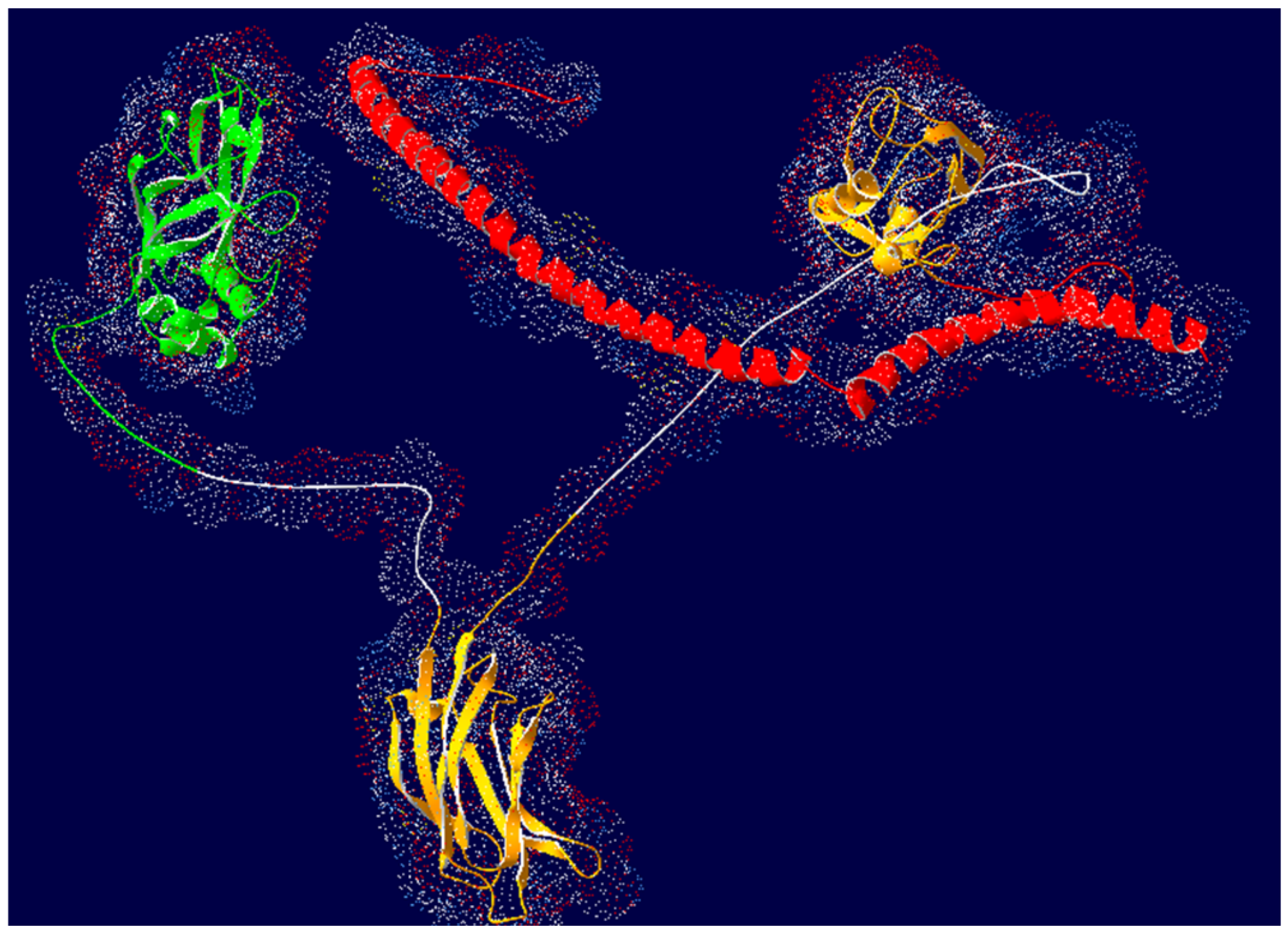

Figure 3. The 3D structure prediction of RPGRIP1L using DeepView. The protein has many disordered regions making it easily accessible for other proteins to interact. Red dots indicate the highly accessible region while white dots are the inaccessible region. Thin strings indicate disordered regions. Three domains are presented in a different colour the N-terminal with two leucine zipper motif (red), the C2 domain (yellow) and RPGR interacting domain (green).

\section{Conclusion and Future Perspectives}

This review has shown the importance of primary cilium in general and RPGRIP1L ciliary transition zone protein in development and diseases. The primary cilium is a cell signalling hub whose functions are determined by RPGRIP1L protein. The RPGRIP1L ensures proper vertebrate development and maintenance by controlling the cilia transition zone's assembly, regulating the ciliary proteasomal activity, and driving cellular autophagy. Through data mining, we have shown that each RPGRIP1L domain plays a vital role in assuring RPGRIP1L's function and proper localization. We postulated that the coiled-coil, C2, and RID domains are responsible for RPGRIP1L centriolar localization, lipid gate formation and protein-protein interaction, respectively. It is also possible that the three domains are not mutually exclusive, particularly in 3D conformation, which allows closer proximity to support interplays between domains. Further experiments on the protein domains and signalling peptide identification will expose the bright potential of RPGRIP1L as a target for future ciliopathy treatment. 


\section{Data availability}

Not applicable in this manuscript.

\section{Conflicts of interest}

The authors have each completed the International Committee of Medical Journal Editors Form for uniform Disclosure of Potential Conflicts of Interest. No authors have any potential conflict of interest to disclose/None of the authors discloses any potential conflict of interest.

\section{Funding statement}

This manuscript is supported by the Indonesia International Institute for Life Sciences Research and Inovation Internal Research Grant.

\section{Acknowledgments}

We would like to thank Cristina Gomez Suarez, Istiqomah Agusta and Chrestella Nathania Denzyl that contributed to the progression of our RPGRIP1L research and make our shared research agenda possible.

\section{References}

[1] N. F. Berbari, A. K. O'Connor, C. J. Haycraft, and B. K. Yoder, "The Primary Cilium as a Complex Signaling Center," Curr. Biol., vol. 19, no. 13, pp. R526-R535, 2009.

[2] S. C. Goetz and K. V Anderson, "The primary cilium: a signalling centre during vertebrate development," Nat. Rev. Genet., vol. 11, no. 5, pp. 331-344, 2010.

[3] G. Wheway, L. Nazlamova, and J. T. Hancock, "Signaling through the Primary Cilium.," Front. cell Dev. Biol., vol. 6, p. 8, 2018.

[4] P. Satir, L. B. Pedersen, and S. T. Christensen, "The primary cilium at a glance," J. Cell Sci., vol. 123, no. 4, pp. 499-503, 2010.

[5] A. M. Waters and P. L. Beales, "Ciliopathies: An expanding disease spectrum," Pediatr. Nephrol., vol. 26, no. 7, pp. 1039-1056, 2011.

[6] L. Wang and B. D. Dynlacht, "The regulation of cilium assembly and disassembly in development and disease," Dev., vol. 145, no. 18, 2018.

[7] K. Szymanska and C. A. Johnson, "The transition zone: An essential functional compartment of cilia," Cilia, vol. 1, no. 1, p. 1, 2012.

[8] A. Dummer, C. Poelma, M. C. DeRuiter, M. J. T. H. Goumans, and B. P. Hierck, "Measuring the primary cilium length: Improved method for unbiased high-throughput analysis," Cilia, vol. 5, no. 1, pp. 1-9, 2016.

[9] P. Satir, "CILIA: before and after," Cilia, vol. 6, no. 1, p. 1, 2017.

[10] H. Lodish, A. Berk, S. L. Zipursky, P. Matsudaira, D. Baltimore, and J. Darnell, "Cilia and flagella: structure and movement," Mol. Cell Biol., 2000.

[11] I. Ibañez-Tallon, N. Heintz, and H. Omran, "To beat or not to beat: roles of cilia in development and disease," Hum. Mol. Genet., vol. 12, no. suppl_1, pp. R27-R35, Apr. 2003.

[12] D. Bray, Cell movements: from molecules to motility. Garland Science, 2000.

[13] A. O. Kovalevskij, Entwickelungsgeschichte des Amphioxus lanceolatus, 11th ed. Memoires de l'Academie Imperiale des Sciences de St.-Petersbourg VII, 1867.

[14] S. Sorokin, "Centrioles and the formation of rudimentary cilia by fibroblasts and smooth muscle cells.," J. Cell Biol., vol. 15, no. 10, pp. 363-377, 1962.

[15] C. A. Poole, M. H. Flint, and B. W. Beaumont, "Analysis of the morphology and function of primary cilia in connective tissues: a cellular cybernetic probe?," Cell Motil., vol. 5, no. 3, pp. 175-193, 1985.

[16] G. Albrecht-Buehler and A. Bushnell, "The ultrastructure of primary cilia in quiescent 3T3 cells," Exp. Cell Res., vol. 126, no. 2, pp. 427-437, 1980.

[17] P. Satir and S. T. Christensen, "Overview of Structure and Function of Mammalian Cilia," Annu. Rev. Physiol., vol. 69, no. 1, pp. 377-400, Feb. 2007.

[18] G. M. W. Adams, B. Huang, G. Piperno, and D. J. L. Luck, "Central-pair microtubular complex of Chlamydomonas flagella: Polypeptide composition as revealed by analysis of mutants," J. Cell Biol., vol. 91, no. 1, pp. 69-76, 1981.

[19] S. O. Ann, "Molecular Motors," Mol. Mot., vol. 422, no. April, pp. 759-765, 2007.

[20] S. Nonaka et al., "Randomisation of left-right asymmetry due to loss of nodal cilia generating leftward flow of 
extraembryonic fluid in mice lacking KIF3B motor protein," Cell, vol. 95, no. 6, pp. 829-837, 1998.

[21] L. E. Ostrowski et al., "A proteomic analysis of human cilia: identification of novel components.," Mol. Cell. Proteomics, vol. 1, no. 6, pp. 451-465, 2002.

[22] S. T. Christensen, C. A. Clement, P. Satir, and L. B. Pedersen, "Primary cilia and coordination of receptor tyrosine kinase (RTK) signalling," J. Pathol., vol. 226, no. 2, pp. 172-184, 2012.

[23] J. L. Badano, N. Mitsuma, P. L. Beales, and N. Katsanis, "The Ciliopathies: An Emerging Class of Human Genetic Disorders," Annu. Rev. Genomics Hum. Genet., vol. 7, no. 1, pp. 125-148, 2006.

[24] M. Adams, "The primary cilium: An orphan organelle finds a home," Nat. Educ., vol. 3, no. 9, p. 54, 2010.

[25] H. Ishikawa, J. Thompson, J. R. Yates, and W. F. Marshall, "Proteomic analysis of mammalian primary cilia," Curr. Biol., vol. 22, no. 5, pp. 414-419, 2012.

[26] V. Singla, "The Primary Cilium as the Cell's Antenna: Signaling at a Sensory Organelle," Science (80-. )., vol. 313, no. 5787, pp. 629-633, Aug. 2006.

[27] K. G. Kozminski, K. A. Johnson, P. Forscher, and J. L. Rosenbaum, "A motility in the eukaryotic flagellum unrelated to flagellar beating.," Proc. Natl. Acad. Sci., vol. 90, no. 12, pp. 5519-5523, 1993.

[28] G. J. Pazour et al., "Chlamydomonas IFT88 and its mouse homologue, polycystic kidney disease gene Tg737, are required for assembly of cilia and flagella," J. Cell Biol., vol. 151, no. 3, pp. 709-718, 2000.

[29] S. Hoyer-Fender, "Centriole maturation and transformation to basal body," Semin. Cell Dev. Biol., vol. 21, no. 2, pp. 142-147, 2010.

[30] J. J. Malicki and C. A. Johnson, "The Cilium: Cellular Antenna and Central Processing Unit," Trends Cell Biol., vol. 27, no. 2, pp. 126-140, 2017.

[31] R. Uzbekov and I. Alieva, "Who are you, subdistal appendages of centriole?," R. Soc. Open Sci., vol. 8, no. 7, 2018.

[32] D. A. Hoey, M. E. Downs, and C. R. Jacobs, "The mechanics of the primary cilium: An intricate structure with complex function," J. Biomech., vol. 45, no. 1, pp. 17-26, Jan. 2012.

[33] A. Benmerah, "The ciliary pocket," Curr. Opin. Cell Biol., vol. 25, no. 1, pp. 78-84, Feb. 2013.

[34] F. R. Garcia-Gonzalo and J. F. Reiter, "Scoring a backstage pass: Mechanisms of ciliogenesis and ciliary access," J. Cell Biol., vol. 197, no. 6, pp. 697-709, 2012.

[35] H. Ishikawa and W. F. Marshall, "Ciliogenesis: Building the cell's antenna," Nat. Rev. Mol. Cell Biol., vol. 12, no. 4, pp. 222-234, 2011.

[36] J. M. Berg, J. L. Tymoczko, and L. Stryer, "Kinesin and dynein move along microtubules," Biochemistry, vol. 5, 2002.

[37] P. G. Czarnecki and J. V. Shah, "The ciliary transition zone: from morphology and molecules to medicine," Trends Cell Biol., vol. 22, no. 4, pp. 201-210, Apr. 2012.

[38] R. G. W. Anderson, "The three-dimensional structure of the basal body from the rhesus monkey oviduct," J. Cell Biol., vol. 54, no. 2, pp. 246-265, 1972.

[39] S. Graser et al., "Cep164, a novel centriole appendage protein required for primary cilium formation," J. Cell Biol., vol. 179, no. 2, pp. 321-330, 2007.

[40] V. S. Gerasimchuk and A. A. Shitov, "The dynamics of domain walls in an easy-plane magnet in the field of an acoustic wave," Phys. Solid State, vol. 45, no. 1, pp. 124-129, 2003.

[41] J. A. Deane, D. G. Cole, E. S. Seeley, D. R. Diener, and J. L. Rosenbaum, "Localisation of intraflagellar transport protein IFT52 identifies basal body transitional fibers as the docking site for IFT particles," Curr. Biol., vol. 11, no. 20, pp. 1586-1590, 2001.

[42] I. R. Gibbons and A. V. Grimstone, "On flagellar structure in certain flagellates.," J. Biophys. Biochem. Cytol., vol. 7, no. 4, pp. 697-716, 1960 .

[43] N. B. Gilula and P. Satir, "The ciliary necklace a ciliary membrane specialisation," J. Cell Biol., vol. 53, no. 2, pp. 494-509, 1972.

[44] B. Craige et al., "CEP290 tethers flagellar transition zone microtubules to the membrane and regulates flagellar protein content," J. Cell Biol., vol. 190, no. 5, pp. 927-940, 2010.

[45] C. L. Williams et al., "MKS and NPHP modules cooperate to establish basal body/transition zone membrane associations and ciliary gate function during ciliogenesis," J. Cell Biol., vol. 192, no. 6, pp. 1023-1041, 2011.

[46] L. Sang et al., "Mapping the NPHP-JBTS-MKS Protein Network Reveals Ciliopathy Disease Genes and Pathways," Cell, vol. 145, no. 4, pp. 513-528, May 2011.

[47] D. Gogendeau et al., "MKS-NPHP module proteins control ciliary shedding at the transition zone," PLoS Biol., vol. 18, no. 3, p. e3000640, 2020.

[48] L. Huang et al., "TMEM237 Is Mutated in Individuals with a Joubert Syndrome Related Disorder and Expands the Role of the TMEM Family at the Ciliary Transition Zone," Am. J. Hum. Genet., vol. 89, no. 6, pp. 713-730, Dec. 2011.

[49] F. R. Garcia-Gonzalo et al., "A transition zone complex regulates mammalian ciliogenesis and ciliary membrane composition," Nat. Genet., vol. 43, no. 8, pp. 776-784, 2011.

[50] S. R. Patnaik, R. K. Raghupathy, X. Zhang, D. Mansfield, and X. Shu, "The Role of RPGR and Its Interacting Proteins in Ciliopathies," J. Ophthalmol., vol. 2015, p. 414781, 2015.

[51] M. Delous et al., "The ciliary gene RPGRIP1L is mutated in cerebello-oculo-renal syndrome (Joubert syndrome 
type B) and Meckel syndrome," Nat. Genet., vol. 39, no. 7, pp. 875-881, 2007.

[52] H. H. Arts et al., "Mutations in the gene encoding the basal body protein RPGRIP1L, a nephrocystin-4 interactor, cause Joubert syndrome," Nat. Genet., vol. 39, no. 7, pp. 882-888, 2007.

[53] C. Gerhardt et al., "The transition zone protein Rpgrip 1 l regulates proteasomal activity at the primary cilium," $J$. Cell Biol., vol. 210, no. 1, pp. 115-133, 2015.

[54] B. Alberts, A. Johnson, J. Lewis, M. Raff, K. Roberts, and P. Walter, "From RNA to protein," in Molecular Biology of the Cell. 4th edition, Garland Science, 2002.

[55] P. Zhou and J. Zhou, "The Primary Cilium as a Therapeutic Target in Ocular Diseases," Front. Pharmacol., vol. 11, 2020.

[56] Y. Zhao et al., "The retinitis pigmentosa GTPase regulator (RPGR)-interacting protein: subserving RPGR function and participating in disk morphogenesis," Proc. Natl. Acad. Sci., vol. 100, no. 7, pp. 3965-3970, 2003.

[57] J. Won et al., "RPGRIP1 is essential for normal rod photoreceptor outer segment elaboration and morphogenesis," Hum. Mol. Genet., vol. 18, no. 22, pp. 4329-4339, 2009.

[58] H. Shiwaku, A. Umino, M. Umino, and T. Nishikawa, "Phencyclidine-induced dysregulation of primary cilia in the rodent brain," Brain Res., vol. 1674, pp. 62-69, Nov. 2017.

[59] Schizophrenia Working Group of the Psychiatric Genomics Consortium, "Biological insights from 108 schizophrenia-associated genetic loci.," Nature, vol. 511, no. 7510, pp. 421-7, Jul. 2014.

[60] M. C. O'Donovan et al., "Identification of loci associated with schizophrenia by genome-wide association and follow-up.," Nat. Genet., vol. 40, no. 9, pp. 1053-5, Sep. 2008.

[61] Bipolar Disorder and Schizophrenia Working Group of the Psychiatric Genomics Consortium. Electronic address: douglas.ruderfer@vanderbilt.edu and Bipolar Disorder and Schizophrenia Working Group of the Psychiatric Genomics Consortium, "Genomic Dissection of Bipolar Disorder and Schizophrenia, Including 28 Subphenotypes.," Cell, vol. 173, no. 7, p. 1705-1715.e16, 2018.

[62] E. Reble, Y. Feng, K. G. Wigg, and C. L. Barr, "DNA Variant in the RPGRIP1L Gene Influences Alternative Splicing," Mol. Neuropsychiatry, vol. 5, no. 1, pp. 97-106, 2019.

[63] A. Mahuzier et al., "Dishevelled stabilisation by the ciliopathy protein Rpgrip1l is essential for planar cell polarity," J. Cell Biol., vol. 198, no. 5, pp. 927-940, 2012.

[64] J. Vierkotten, R. Dildrop, T. Peters, B. Wang, and U. Rüther, "Ftm is a novel basal body protein of cilia involved in Shh signalling," Development, vol. 134, no. 14, pp. 2569-2577, 2007.

[65] A. R. Jauregui, K. C. Q. Nguyen, D. H. Hall, and M. M. Barr, "The Caenorhabditis elegans nephrocystins act as global modifiers of cilium structure," J. Cell Biol., vol. 180, no. 5, pp. 973-988, 2008.

[66] N. T. Gorden et al., "CC2D2A is mutated in Joubert syndrome and interacts with the ciliopathy-associated basal body protein CEP290," Am. J. Hum. Genet., vol. 83, no. 5, pp. 559-571, 2008.

[67] B. Chih et al., "A ciliopathy complex at the transition zone protects the cilia as a privileged membrane domain," Nat. Cell Biol., vol. 14, no. 1, pp. 61-72, 2012.

[68] U. Mayer et al., "The proteome of rat olfactory sensory cilia," Proteomics, vol. 9, no. 2, pp. 322-334, 2009.

[69] G. J. Pazour, N. Agrin, J. Leszyk, and G. B. Witman, "Proteomic analysis of a eukaryotic cilium," J. Cell Biol., vol. 170, no. 1, pp. 103-113, 2005.

[70] V. L. Jensen et al., "Formation of the transition zone by Mks5/Rpgrip1L establishes a ciliary zone of exclusion (CIZE) that compartmentalises ciliary signalling proteins and controls PIP2 ciliary abundance," EMBO J., vol. 34 , no. 20, pp. 2537-2556, Oct. 2015.

[71] A. Wiegering et al., "Cell type-specific regulation of ciliary transition zone assembly in vertebrates," EMBO J., vol. 37, no. 10, p. e97791, May 2018.

[72] Y. P. Liu et al., "Ciliopathy proteins regulate paracrine signaling by modulating proteasomal degradation of mediators," J. Clin. Invest., vol. 124, no. 5, pp. 2059-2070, 2014.

[73] B. Wang, J. F. Fallon, and P. A. Beachy, "Hedgehog-regulated processing of Gli3 produces an anterior/posterior repressor gradient in the developing vertebrate limb," Cell, vol. 100, no. 4, pp. 423-434, 2000.

[74] C. Gerhardt, J. M. Lier, S. Kuschel, and U. Rüther, "The ciliary protein Ftm is required for ventricular wall and septal development," PLoS One, vol. 8, no. 2, p. e57545, 2013.

[75] L. Besse et al., "Primary cilia control telencephalic patterning and morphogenesis via Gli3 proteolytic processing," Development, vol. 138, no. 10, pp. 2079-2088, 2011.

[76] A. Inoko et al., "Trichoplein and Aurora A block aberrant primary cilia assembly in proliferating cells," J. Cell Biol., vol. 197, no. 3, pp. 391-405, 2012.

[77] K. Kasahara et al., "Ubiquitin-proteasome system controls ciliogenesis at the initial step of axoneme extension," Nat. Commun., vol. 5, pp. 1-10, 2014.

[78] Z. Tang et al., "Autophagy promotes primary ciliogenesis by removing OFD1 from centriolar satellites," Nature, vol. 502, no. 7470, pp. 254-257, 2013.

[79] O. Pampliega et al., "Functional interaction between autophagy and ciliogenesis," Nature, vol. 502, no. 7470, pp. 194-200, 2013.

[80] A. Struchtrup, A. Wiegering, B. Stork, U. Rüther, and C. Gerhardt, "The ciliary protein RPGRIP1L governs autophagy independently of its proteasome-regulating function at the ciliary base in mouse embryonic fibroblasts," Autophagy, vol. 14, no. 4, pp. 567-583, 2018. 
[81] T. P. Neufeld, "TOR-dependent control of autophagy: biting the hand that feeds," Curr. Opin. Cell Biol., vol. 22, no. 2, pp. 157-168, Apr. 2010.

[82] C. H. Jung, S. H. Ro, J. Cao, N. M. Otto, and D. H. Kim, "MTOR regulation of autophagy," FEBS Lett., vol. 584, no. 7, pp. 1287-1295, 2010.

[83] S. Wang, M. J. Livingston, Y. Su, and Z. Dong, "Reciprocal regulation of cilia and autophagy via the MTOR and proteasome pathways," Autophagy, vol. 11, no. 4, pp. 607-616, 2015. 